\title{
The worm turns on Helicobacter pylori
}

Fox JG, Beck P, Dangler CA, et al. Concurrent enteric helminth infection modulates inflammation and gastric immune responses and reduces helicobacterinduced gastric atrophy. Nat Med 2000;6:536-42.

\begin{abstract}
Helicobacter pylori is causally associated with gastritis and gastric cancer. Some developing countries with a high prevalence of infection have high gastric cancer rates, whereas in others, these rates are low. The progression of helicobacterinduced gastritis and gastric atrophy mediated by type 1 T-helper cells may be modulated by concurrent parasitic infection. Here, in mice with concurrent helminth infection, helicobacterassociated gastric atrophy was reduced considerably despite chronic inflammation and high helicobacter colonization. This correlated with a substantial reduction in mRNA for cytokines and chemokines associated with a gastric inflammatory response of type 1 T-helper cells. Thus, concurrent enteric helminth infection can attenuate gastric atrophy, a premalignant lesion.
\end{abstract}

\section{Comment}

This study uses Helicobacter felis infection of C57BL/6 mice as a model of Helicobacter pylori infection in humans. Infection with $H$ felis produces gastritis and gastric atrophy with mucous hyperplasia, metaplasia, and parietal cell loss. However, in mice preinfected with a nematode parasite Heligmosomoides polygyrus there was reduced parietal cell loss and less mucous cell hypertrophy and metaplasia. Measurement of gastric mucosal cytokines revealed that preinfection with $H$ polygyrus reduced the number of transcripts for Th1 associated cytokines and chemokines but increased transcripts for Th2-type cytokines. The authors suggest that concurrent $\mathrm{Th} 2 \mathrm{immune}$ responses elicited by the nematode downregulate the tissue damaging Th1 response elicited by $H$ felis. In addition, the authors speculate that different rates of helminthic infection in different parts of the world might explain why, despite equally poor socioeconomic conditions and high rates of infection with $H$ pylori, there are major differences in the incidence of gastric cancer.

However, before gastroenterologists recommend sending all their patients on a tropical holiday and suggest they eat all the unwashed and uncooked vegetables they can find, a few words of caution are needed. While this is an intriguing study, it is fair to say that the authors highlighted the positive aspects of their results and did not dwell on the negative aspects. The mice were studied at two time points, eight and 16 weeks after $H$ felis infection. At eight weeks, although chronic submucosal inflammation and parietal cell loss were decreased in the worm infected animals, there was no decrease in mucosal chronic inflammation and in fact the worm infected animals had more severe gastritis. At 16 weeks the reduction in chronic submucosal inflammation was not seen, suggesting it was a chance finding. Similarly, the increased active gastritis in the nonworm infected mice was not seen at 16 weeks, again probably a chance finding. In contrast, hyperplasia, parietal cell loss, and mucous cell hypertrophy and metaplasia were significantly decreased in the worm infected animals compared with non-worm infected mice. Strikingly, whereas the control mice had eliminated $H$ felis by 16 weeks, there was still substantial bacterial colonisation in the worm infected animals.

It is also worth commenting on the quality of the data. Even in the indices where there were reductions in the histological scores in the worm infected mice, there was always considerable overlap between the groups. None of the $\mathrm{p}$ values were $<0.01$ and all disappeared if a Bonferroni correction was performed.

Semiquantitative reverse transcriptase-polymerase chain reaction was used to assess cytokine $\mathrm{mRNA}$ concentrations in the stomachs of all mice. While this is not an ideal method, the fact that there was a consistent downward trend for nearly all the cytokines usually associated with inflammation is encouraging. RNAse protection assays were used to examine chemokine expression and again the downward trend in the worm infected animals is impressive.

It is now clear in humans and certain strains of mice that the $\mathrm{CD} 4^{+} \mathrm{T}$ cells which infiltrate the stomach during $H$ pylori infection are of a polarised Th1 phenotype-that is, secrete interferon $\gamma$, interleukin 2 , and tumour necrosis factor $\alpha .^{1-3}$ Mouse experiments have clearly shown that in the absence of an immune system or the ability to make interferon $\gamma, H$ pylori infection elicits minimal pathological changes. ${ }^{45}$ Thus in common with many other gut diseases (parasitic worm infections, coeliac disease, Crohn's disease $^{6}$ ) the inflammation is due to the host $T$ cell response. It is also well established that one of the major factors which controls the differentiation of cells along the Th1 or Th2 pathway is the cytokine environment during the initial stages of the response. Thus during bacterial infection, macrophages can secrete interleukin 12 which binds to its specific receptor on the $\mathrm{T}$ cell which results in phosphorylation of STAT4. STAT4 moves to the nucleus and interacts with the interferon $\gamma$ promoter driving the cell along the Th1 pathway. In contrast, interleukin 4 produced by mast cells during the initial phases drives cells along a Th2 pathway by activating the STAT6 pathway. ${ }^{7}$

Thus in the study of Fox et al it is assumed that the worm infection, which is known to elicit a Th2 response in the gut, ${ }^{8}$ biases the anti- $H$ felis $\mathrm{T}$ cell response along the same pathway. Indeed, the gastric mucosa of worm infected $H$ felis infected mice contained greater amounts of mRNA for interleukin 10, interleukin 4, and transforming growth factor $\beta$. It would however be premature to come to this conclusion because $H$ polygyrus is an unusual parasite. It has been known for a long time that $H$ polygyrus secrete immunomodulatory proteins so that infected animals are generally immunosuppressed. ${ }^{9}$ Thus the reduction in gastric inflammation may be due to this 
non-specific attenuation of the Th1 anti- $H$ felis response rather than immune deviation. The increased Th2 cytokines in the stomach wall of the worm infected mice could be due to migration into the inflamed gastric mucosa of Th2 cells responding to $H$ polygyrus antigens.

Even if the reduction in inflammation was due to induction of a Th2 anti- $H$ felis response, this may not be a desirable outcome, especially as the stomachs of these animals contain large numbers of bacteria. There is a misconception that $\mathrm{Th} 2$ responses in the gut are non-inflammatory, helping to drive the IgA response. However, this is clearly not the case. Another nematode, Trichinella spiralis, elicits a large Th2 response in the small intestine accompanied by extremely high concentrations of proinflammatory cytokines and extensive mucosal injury. ${ }^{10}$ It would be interesting to determine if this occurs in the long term in mice coinfected with $H$ felis and $H$ polygyrus.

Finally, does this paper shed any light on the African paradox - that is, despite the high incidence of $H$ pylori, there is a low incidence of gastric cancer whereas in South America despite an equally high incidence of $H$ pylori, gastric cancer is common? The authors suggest that because subjects with $H$ pylori in South America live at higher altitudes where there may be a lower incidence of helminth infection, this may influence the development of
$H$ pylori associated cancer. It is worth pointing out that there are also mountains in Africa, some quite big ones in fact.

T T MACDONALD

Centre for Infection, Allergy, Inflammation and Repair,

University of Southampton School of Medicine,

Southampton General Hospital,

Southampton SO16 6YD, UK

t.t.macdonald@soton.ac.uk

1 Karttunnen R, Karttunnen T, Ekre H-P T, et al. Interferon-gamma and interleukin-4 secreting cells in the gastric antrum in Helicobacter pylori associated gastritis. Gut 1995;36:341-5.

2 Bamford KB, Fan X, Crowe SE, et al. Lymphocytes in the human gastric mucosa during Helicobacter pylori infection have a T helper cell 1 phenomucosa during Helicobacter pylori infection

3 Smythies LE, Waites KB, Lindsey JR, et al. Helicobacter pylori-induced mucosal inflammation is Th1 mediated and exacerbated in IL-4, but not mucosal inflammation is Thl mediated and exacerbated in II

4 Sawai N, Kita M, Kodama T, et al. Role of gamma interferon in Helicobacter pylori-induced gastric inflammatory responses in a mouse model. Infect Immunol 1999;67:279-85.

5 Eaton KA, Ringler SR, Danon SJ. Murine splenocytes induce severe gastritis and delayed type hypersensitivity and suppress bacterial colonization in Helicobacter pylori-infected SCID mice. Infect Immunol 1999;67:4594-602.

6 MacDonald TT. Effector and regulatory lymphoid cells and cytokines in mucosal sites. Curr Top Microbiol Immunol 1999;236:113-25.

7 Wurster AL, Tanaka T, Grusby MJ. The biology of STAT4 and STAT6. Oncogene 2000;15:2577-84.

8 Svetic A, Madden KB, Zhou Xd, et al. A primary intestinal helminthic infection rapidly induces a gut-associated elevation of Th2-associated infection rapidly induces a gut-associated elevation

9 Pritchard DI, Lawrence CE, Appleby P, et al. Immunosuppressive proteins secreted by the gastrointestinal nematode parasite Heligmosomoides polygyrus. Int 7 Parasitol 1994;24:495-50.

10 Lawrence CE, Paterson JCM, Higgins LM, et al. IL-4 regulated enteropathy in an intestinal nematode infection. Eur f Immunol 1998;28:2672-84. 